\title{
SIMULATION AND THEORY OF ABNORMAL GRAIN GROWTH-ANISOTROPIC GRAIN BOUNDARY ENERGIES AND MOBILITIES
}

\author{
A. D. ROLLETT, ${ }^{1}$ D. J. SROLOVITZ ${ }^{2}$ and M. P. ANDERSON ${ }^{3}$ \\ 'Los Alamos National Laboratory, Los Alamos, NM 87545, \\ ${ }^{2}$ Department of Materials Science and Engineering, University of Michigan, Ann Arbor, MI 48109 and \\ ${ }^{3}$ Corporate Research Science Laboratory, Exxon Research and Engineering Company, Annandale, \\ NJ 08801, U.S.A.
}

(Received 23 March 1988)

\begin{abstract}
Abnormal grain growth has been studied by means of a computer-based Monte Carlo model. This model has previously been shown to reproduce many of the essential features of normal grain growth. The simulations presented in this work are based on a modified model in which two distinct types of grains are present. These two grain types might correspond to two components of different crystallographic orientation, for example. This results in three classes of grain boundaries: (a) between unlike types, (b) between grains of the first type and (c) between grains of the second type, to which different grain boundary energies or different mobilities can be assigned. Most simulations started with a single grain of the first type embedded in a matrix of grains of the second type. Anisotropic grain boundary energies were modeled by assigning a higher energy to boundaries between like type than to boundaries between grains of unlike type. For this case, abnormal grain growth only occurred for an energy ratio greater than 2 and then wetting of the matrix by the abnormal grain occurred. Anisotropic grain boundary mobilities were modeled by assigning a lower mobility to boundaries between grains of like type than to boundaries between unlike type. For this case the extent of abnormal grain growth varied with the ratio of mobilities and it is tentatively concluded that there is a limiting ratio of size of the abnormal grain relative to the matrix. A simple treatment of anisotropic grain boundary mobility was developed by modifying Hillert's grain growth model [Acta metall. 13, 227 (1965)]. This theoretical treatment also produced a limiting ratio of relative size that is a simple function of the mobility ratio.
\end{abstract}

Résumé-La croissance anormale des grains a été étudiée au moyen d'une simulation de Monte Carlo sur un ordinateur. Ce modèle s'est déjà révélế capable de reproduire beaucoup des caractéristiques essentielles de la croissance normale de grain. Les simulations présentées dans cet article sont basées sur un modèle modifié dans lequel deux types distincts de grains sont présents. Ces deux types de grains pourraient correspondre à deux composantes d'orientations cristallographiques différentes, par exemple. Il en résulte trois classes de joints de grains: (a) entre types de grains différents; (b) entre grains du premier type; (c) entre grains due second type, auxquels peuvent être assignées différentes énergies intergranulaires ou différentes mobilités. La plupart des simulations concernent un seul grain du premier type entouré par une matrice de grains du second type. On a tenu compte des énergies intergranulaires anisotropes en assignment aux joints séparant des grains du même type une énergie plus grande qu'aux joints séparant des grains de types différents. Dans ce cas, la croissance anormale des grains se produit seulement pour un rapport d'énergies supérieur à 2 et l'on observe ensuite un mouillage de la matrice par le grain anormal. On a modélisé les mobilités anisotropes des joints de grains en assignant aux joints séparant des grains de même type une mobilité plus faible qu'aux joints séparant des grains de types différents. Dans ce cas, l'importance de la croissance anormale de grain varie avec le rapport des mobilités et il semble qu'il y ait un rapport limite entre la taille du grain anormal et celle des grains de matrice. Un traitement simple de la mobilité anisotrope des joints de grains a été développé en modifiant le modèle de croissance de Hilbert [Acta metall. 13, 227 (1965)]. Ce traitement théorique a aussi donné une valeur limite de taille relative qui est une fonction simple du rapport des mobilités.

Zusammenfassung-Das anomale Kornwachstum wurde mit einer Monte Carlo-Simulation im Rechner untersucht. Früher wurde gezeigt, daß das benutzte Modell viele der wesentlichen Eigenschaften des normalen Kornwachstums wiedergibt. Die in dieser Arbeit dargestellten Simulationen beruhen auf einem modifizierten Modell, bei dem zwei unterschiedliche Korntypen vorhanden sind. Diese beiden Korntypen können zum Beispiel zwei Typen mit unterschiedlicher Kristallorientierung entsprechen. Diese Bedingung führt zu drei Klassen von Korngrenzen, solchen zwischen ungleichen Körnern und solchen ausschließlich zwischen Körnern des ersten und des zweiten Typs; entsprechend können diese Korngrenzarten unterschiedliche Energien und Beweglichkeiten aufweisen. Die meisten der Simulationen begannen mit einem einzigen Korn des ersten Typs, welches in einer Matrix von Körnern des zweiten Typs eingebettet war. Anisotrope Korngrenzenergien wurden im Modell beschriehen, indem den Korngrenzen zwischen gleichen Körnern eine höhere Energie als zwischen ungleichen Körnern zugeschrieben wurde. In diesem Fall ergab sich anormales Kornwachstum nur, wenn das Verhältnis der Energien größer als 2 war und dann das anormale Korn die Matrix benetzte. Anisotrope Korngrenzbeweglichkeiten wurden im Modell beschrieben, indem Korngrenzen zwischen Körnern des gleichen Typs mit einer geringeren Beweglichkeit als zwischen ungleichen Körnern versehen wurden. In diesem Fall hing der Grad des anomalen Wachstums von dem Verhältnis der Beweglichkeiten ab; daraus kann gefolgert werden, daB es eine Grenze 
im Verhältnis der Größe des anormalen Kornes zu dem dér Matrix gibt. Mit einer Modifikation des Kornwachstumsmodelles von Hillert [Acta metall. 13, 227 (1965)] wurde eine einfache Behandiung des anisotropen Korngrenzbeweglichkeit ermöglicht. Diese theoretische Behandlung ergab ebenfalls eine Grenze in der Größe der relativen Korngröße, diese Grenze ist eine einfache Funktion des Verhältnisses der Beweglichkeiten.

\section{INTRODUCTION}

Normal grain growth is the phenomenon of coarsening of the grain structure of polycrystalline metals and ceramics. The kinetics of normal grain growth can be characterized by a proportionality between the mean grain radius and $t^{n}$, where $t$ is time and $n$ is the grain growth exponent. Experimentally, $n$ is known to lie between 0 and $0.5[1-3]$. Most theories $[4,5]$ and recent computer simulations [6] show that $n=0.5$ in the long time limit. However, when diffusing impurities are present, exponents less than 0.5 are expected $[7,8]$. The other notable characteristic of normal grain growth is an invariance of the grain size distribution (i.e. grain size normalized by the mean grain size) with time [9]. Abnormal grain growth, however, leads to a grain size distribution that does vary with time. The cause of this lack of invariance is the growth of a minority of "abnormal" grains into the matrix of "normal" grains. This microstructural characteristic is the same as for primary recrystallization which has led to the term secondary recrystallization. Unlike in grain growth where the driving force is related to the reduction in grain boundary area, the driving force for primary recrystallization is the stored energy from prior plastic deformation. Previous attempts to simulate abnormal grain growth [10] were only successful for the case where the normal grains were modeled (in two dimensions) as having a higher surface energy (uniform over the area of each grain) than the abnormal grains. The assumptions of this model and its results are exactly equivalent to those for primary recrystallization, as shown in subsequent simulations [11]. The work presented here shows, however, that texture effects can play an important role in this phenomenon.

The experimental literature clearly shows that abnormal grain growth can occur in pure metals $[12,13]$. Investigations on iron [13-16] established that normal grain growth in the matrix was inhibited by prior straining. In work on $\mathrm{Pb}$ and $\mathrm{Cd}$ alloys [12] both texture inhibition and strain inhibition of normal grain growth were suggested as possible reasons for abnormal grain growth. Texture inhibition of normal grain growth is based on the idea that if the normal grain structure is strongly textured, the grain boundaries will be low angle boundaries and therefore have low mobility. The abnormal grains, on the other hand, are assumed to have sufficiently different crystallographic orientations (relative to the normal matrix grains) such that their boundaries are all high angle, high mobility grain boundaries. Some circumstantial evidence for the influence of texture in abnormal grain growth can be found in the work of
Petrovic and Ebert on thoriated nickel $[17,18]$. They found that abnormal grain growth occurred and led to changes in the crystallographic orientation distribution of the polycrystalline $\mathrm{Ni}$ alloy. A firmer link between texture and abnormal grain growth has been made by Harase and co-workers. In a $\mathrm{Fe}-50 \mathrm{Ni}$ alloy they found [19] that the largest grains after secondary recrystallization had a high frequency of coincidence boundaries such as $\Sigma 7$ or $\Sigma 19 b$ with the primary matrix grains. Additional studies have shown that abnormal grain growth can occur because normal grain growth in the matrix is restricted by a distribution of second phase particles. May and Turnbull [20] demonstrated this effect in the commercially important material, silicon-iron where the occurrence of abnormal grain growth depended on the presence of $\mathrm{MnS}$ particles. These experimental studies indicate that the abnormal grain growth can occur when the boundaries between abnormal and normal grains are much higher mobility than between normal or matrix grains.

In the field of primary recrystallization, the effects of grain boundary mobility on the texture of the material is well established. Primary recrystallization is closely related to abnormal grain growth in that it involves certain (new) grains growing through and consuming an existing polycrystalline matrix. The theory of Oriented Growth [e.g. Ref. 21] states that there are certain orientation relationships between grains that have a higher mobility than that for general grain misorientations. The misorientation that is most commonly claimed to have high mobility in experiments on face centered cubic materials is that of a $38^{\circ}$ rotation about a $\langle 111\rangle$ axis. This effect is illustrated by data from Aust and Rutter [22], Fig. 1, who demonstrated the existence of large differences in grain boundary mobility as a function of misorientation. Abbruzzese and Lucke [23, 24] have recently developed a theory of grain growth that accounts for variations in mobility between classes of grains.

Varying the crystallographic misorientation across a grain boundary is also expected to change the grain boundary energy. For small misorientations Read and Shockley [25] showed that the grain boundary energy varies with misorientation. Such simple relationships are reasonable for misorientations up to of order $10^{\circ}$ and the effects of incorporating such low-angle boundaries into a computer model were explored by Grest et al. [26]. These simulations rarely produced a large grain with high angle boundaries whose size increased faster than the mean. The focus of that study, however, was on the grain size distribution and the kinetics of normal grain growth and did not explore the possibilities for abnormal grain 


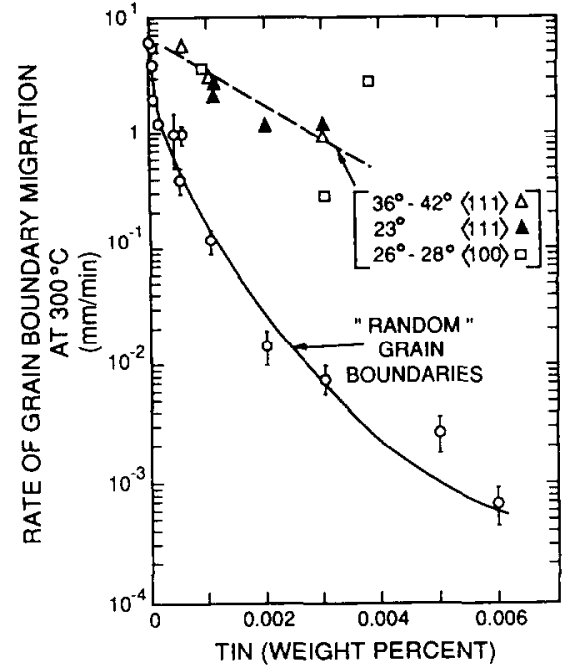

Fig. 1. Plot of grain boundary migration rate vs tin content of $\mathrm{Pb}$ alloys for special orientation relationships and general boundaries, from Aust and Rutter [22]

growth. One consequence of the fact that the grain boundary energy is dependent on the crystallographic misorientation is that the grain boundary velocity is a function of the crystallographic misorientation. (The velocity of a boundary is generally assumed to be proportional to its mobility and curvature [27].)

In this paper we present a series of simulations on the effects of anisotropic grain boundary mobilities and energies on abnormal growth. Since both of these effects can be attributed to crystallographic texture, we present our results in that light. However, these results are not limited to texture effects since anisotropy can be introduced in a number of ways. Section 2 of this paper presents the simulation technique employed and the results of simulations based on the notion of abnormal grain growth due to anisotropy in grain boundary energy. It is found that abnormal grain growth can occur as a result of this anisotropy; however, the microstructures produced are not in agreement with those generally observed. The effects of anisotropic grain boundary mobility on abnormal grain growth is the subject of Section 3. In this case, the simulations yield microstructures and kinetics which are in excellent agreement with experimental observations of abnormal grain growth. An analytical description of anisotropic grain boundary mobility induced abnormal grain growth in the framework of Hillert's [4] grain growth model is presented in Section 4. Finally, in the last section, we discuss the consequences of these results.

\section{ANISOTROPIC GRAIN BOUNDARY ENERGIES}

In the following simulations, we assume that the initial microstructure has a strong crystallographic texture. Experimental studies on $\mathrm{Ni}[28]$ and $\mathrm{Al}$ [29] indicate that abnormal growth in textured materials leads to a marked change in texture. In certain cases,

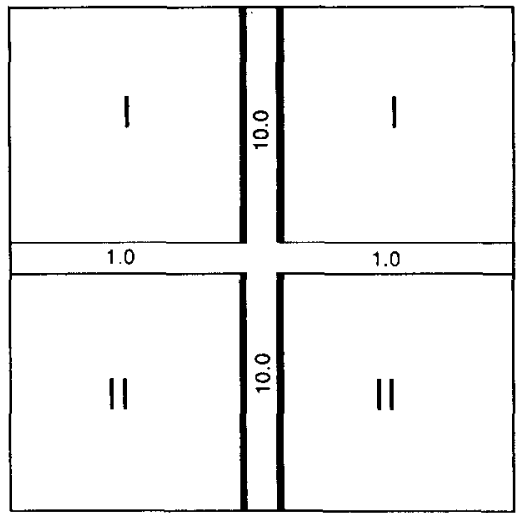

Fig. 2. Diagram of grain boundary energies with high energy boundaries between grains of unlike type.

the growth of a minority texture component is seen to grow and dominate the structure. In the present study, the simplifying assumption is made that all grains correspond to one of two possible texture components (Type I or Type II). Two possibilitics arc examined for the dependence of grain boundary energy on misorientation between grains. The first is illustrated in Fig. 2 which shows an arrangement where the energy between grains of like type (I-I or II-II boundary) is high but the energy between grains of unlike type (I-II boundary) is low. Figure 3 shows that if the ratio of the two energies is large enough, the small, four-sided Type I grain that would normally shrink and vanish, will grow. One could equally well invert the type labels and show that isolated Type II grains should also grow. This type of minority growth corresponds to the experimental results alluded to earlier in this paragraph. The second possibility is where only Type II-Type II boundaries have high energy; this is used to explore the kinetics of abnormal grain growth. While the assumptions about the nature of the texture and the resultant grain

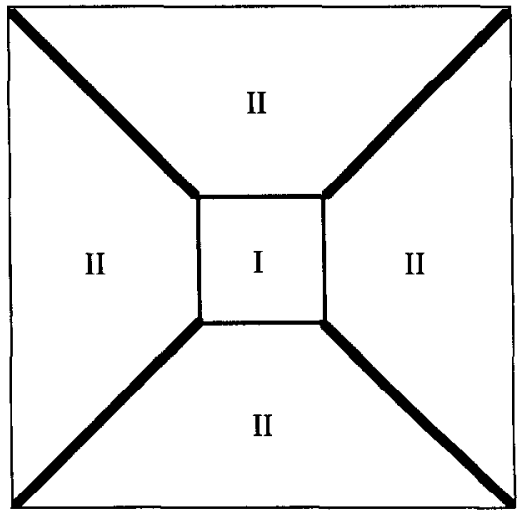

Fig. 3. Diagram showing how the arrangement of grain boundary energies illustrated in the previous figure can cause a small, four-sided grain, surrounded by grains of unlike type, to grow. Under isotropic boundary energy conditions, such a grain would shrink and vanish. 
boundaries is idealized, it provides a simple description of the types of phenomena which can result.

A Monte Carlo model was used to simulate grain growth under the same conditions used previously for normal grain growth $[30,31]$. A two dimensional triangular lattice of 200 by 200 sites was employed. The simulations were preformed with 48 possible orientations at any lattice site (i.e. $Q=48$ ) and a parameter $C$ was established to distinguish between Type I and Type II orientations. Given that the orientation at the $i$ th site is $S_{i}$, if $S_{i} \leqslant C$ then the grain is Type I whereas if $S_{i}>C$, the grain is Type II. The energetics that describe the interaction between lattice sites was defined as follows.

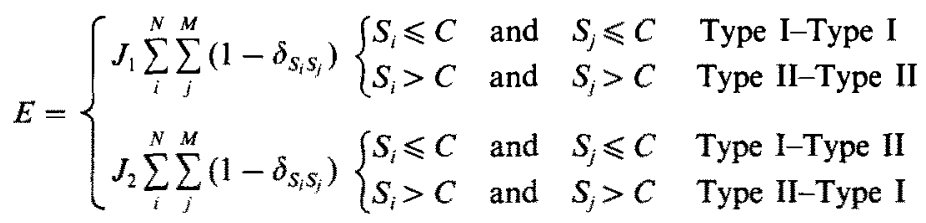

where $J_{1}$ and $J_{2}$ are positive constants such that $J_{1}>J_{2}$, the summation on $i$ is over all $N$ sites and the summation on $j$ is over the $M$ nearest neighbors of the $i$ th site. The choice of $C$ governs the initial fraction of Type I vs Type II grain orientations.

A simulation was performed with $C=7$ and $Q=48$ so that the initial area fraction of Type $I$ grains was approximately 0.15 . The initial microstructure was obtained by simulating normal grain growth for $1000 \mathrm{MCS}$, at which point the system had on the order of 1000 grains [30, 31]. The simulations with the ratio of high to low energies set at 10 (i.e. $J_{1} / J_{2}=10$ ) produced a microstructure consisting of a mosaic arrangement of alternating grains of Type I and Type II, Fig. 4. This results from an elimination of high energy Type I-Type II grain boundary segments. Coarsening of the resultant mosaic structure was slow, compared to that in normal grain growth, because of the large "activation energy barrier" associated with having to form lengths of high energy boundary during the elimination of individual grains. These choices of grain boundary energies did not yield any noticeable abnormal growth.

Despite the fact that the conditions employed above did not lead to abnormal growth, the model of anisotropic grain boundary energies exhibits abnormal grain growth in another limit. A simulation was performed using the same arrangement of grain boundary energies as was shown in Fig. 2 but with the Type I-Type I energy set to the same low value as for the Type I-Type II boundaries, Fig. 5. The energetics for this arrangement of grain boundary energies is determined by

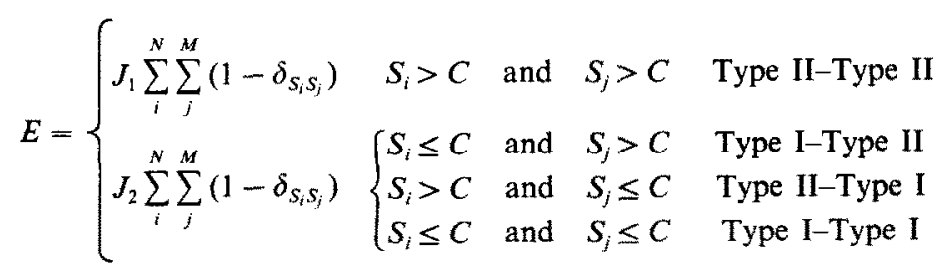

eliminated. Figure 6 shows that the Type I grains (unshaded), initially in the minority, grow at the expense of the Type II (shaded) grains and eventually dominate the microstructure. Type II grains that become isolated shrink and disappear rapidly whereas those at triple points do so more slowly. The microstructures bear a strong resemblance to those observed during abnormal grain growth. If, as suggested above Types I and II are associated with different texture components, such abnormal growth produces major textural modification because a minority component becomes the majority component.

In order to visualize this type of abnormal grain growth phenomena more clearly, simulations were performed where a single grain was made Type I with the arrangement of energies shown in Fig. 5 and all other grains were Type II. In detail, the orientation numbers of all the grains were incremented by one and a new, circular grain was inserted in the structure with $S=1$. The parameter $C$ was set to 1 such that the circular grain was made abnormal and $Q=49$. The results of this type of simulation with $J_{1} / J_{2}=10$ is shown in Fig. 7. Clearly, the large grain, Type I, undergoes abnormal grain growth as indicated by its dramatic increase in size relative to the matrix grains. Interestingly, the abnormal grain wets the matrix grain boundaries. As a consequence, individual matrix grains are separated from their neighboring matrix grains by the abnormal growth front and become embedded in the growing abnormal grain. These isolated matrix grains then gradually 

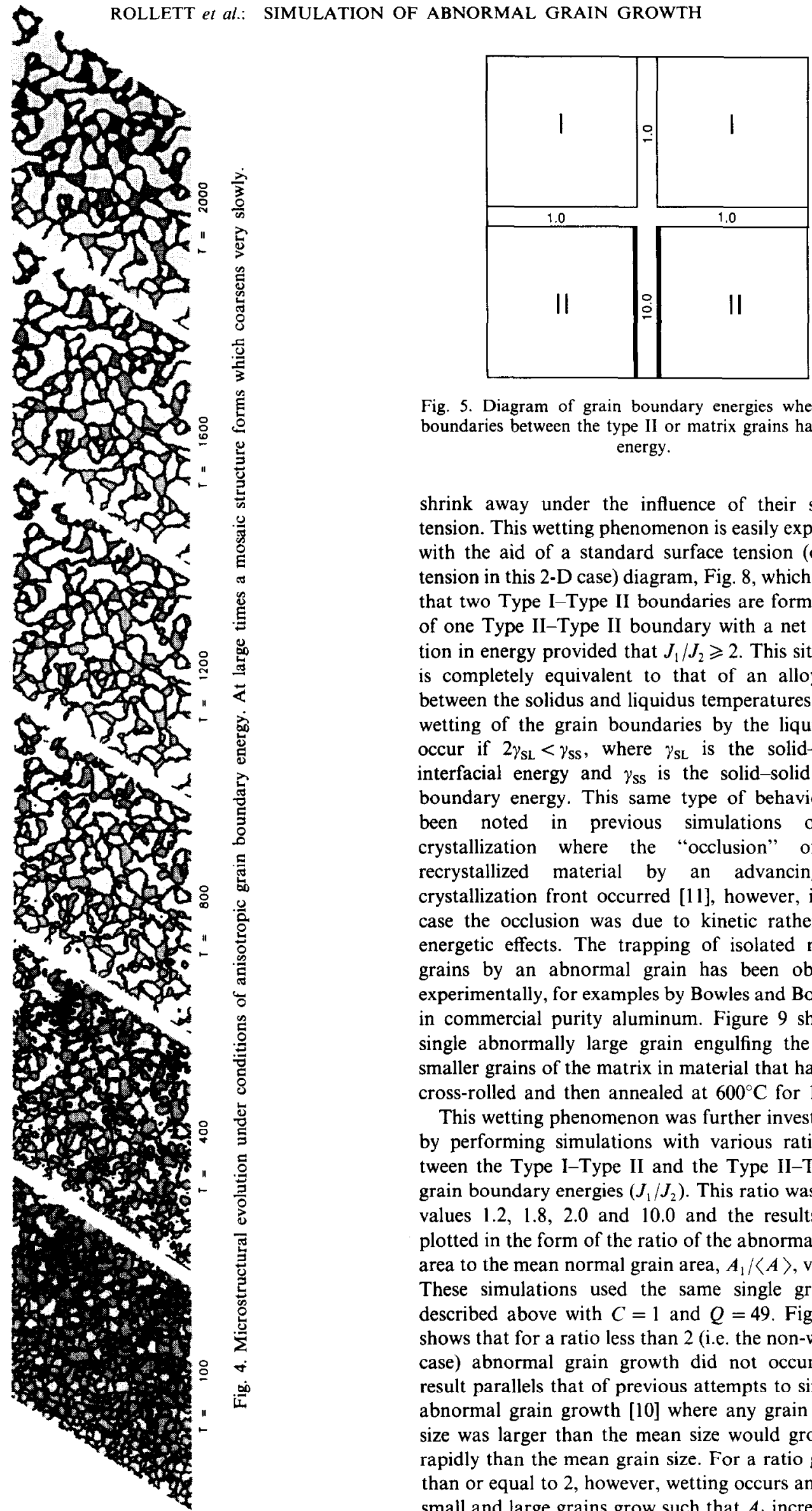

Fig. 5. Diagram of grain boundary energies where only boundaries between the type Il or matrix grains have high energy.

shrink away under the influence of their surface tension. This wetting phenomenon is easily explicable with the aid of a standard surface tension (or line tension in this 2-D case) diagram, Fig. 8, which shows that two Type I Type II boundaries are formed out of one Type II-Type II boundary with a net reduction in energy provided that $J_{1} / J_{2} \geqslant 2$. This situation is completely equivalent to that of an alloy held between the solidus and liquidus temperatures where wetting of the grain boundaries by the liquid can occur if $2 \gamma_{\mathrm{SL}}<\gamma_{\mathrm{SS}}$, where $\gamma_{\mathrm{SL}}$ is the solid-liquid interfacial energy and $\gamma_{\mathrm{ss}}$ is the solid-solid grain boundary energy. This same type of behavior has been noted in previous simulations of recrystallization where the "occlusion" of unrecrystallized material by an advancing recrystallization front occurred [11], however, in that case the occlusion was due to kinetic rather than energetic effects. The trapping of isolated normal grains by an abnormal grain has been observed experimentally, for examples by Bowles and Boas [32] in commercial purity aluminum. Figure 9 shows a single abnormally large grain engulfing the much smaller grains of the matrix in material that has been cross-rolled and then annealed at $600^{\circ} \mathrm{C}$ for $1 \mathrm{~h}$.

This wetting phenomenon was further investigated by performing simulations with various ratios between the Type I-Type II and the Type II-Type II grain boundary energies $\left(J_{1} / J_{2}\right)$. This ratio was given values 1.2, 1.8, 2.0 and 10.0 and the results were plotted in the form of the ratio of the abnormal grain area to the mean normal grain area, $A_{1} /\langle A\rangle$, vs time. These simulations used the same single grain as described above with $C=1$ and $Q=49$. Figure 10 shows that for a ratio less than 2 (i.e. the non-wetting case) abnormal grain growth did not occur. This result parallels that of previous attempts to simulate abnormal grain growth [10] where any grain whose size was larger than the mean size would grow less rapidly than the mean grain size. For a ratio greater than or equal to 2 , however, wetting occurs and both small and large grains grow such that $A_{1}$ increases as 

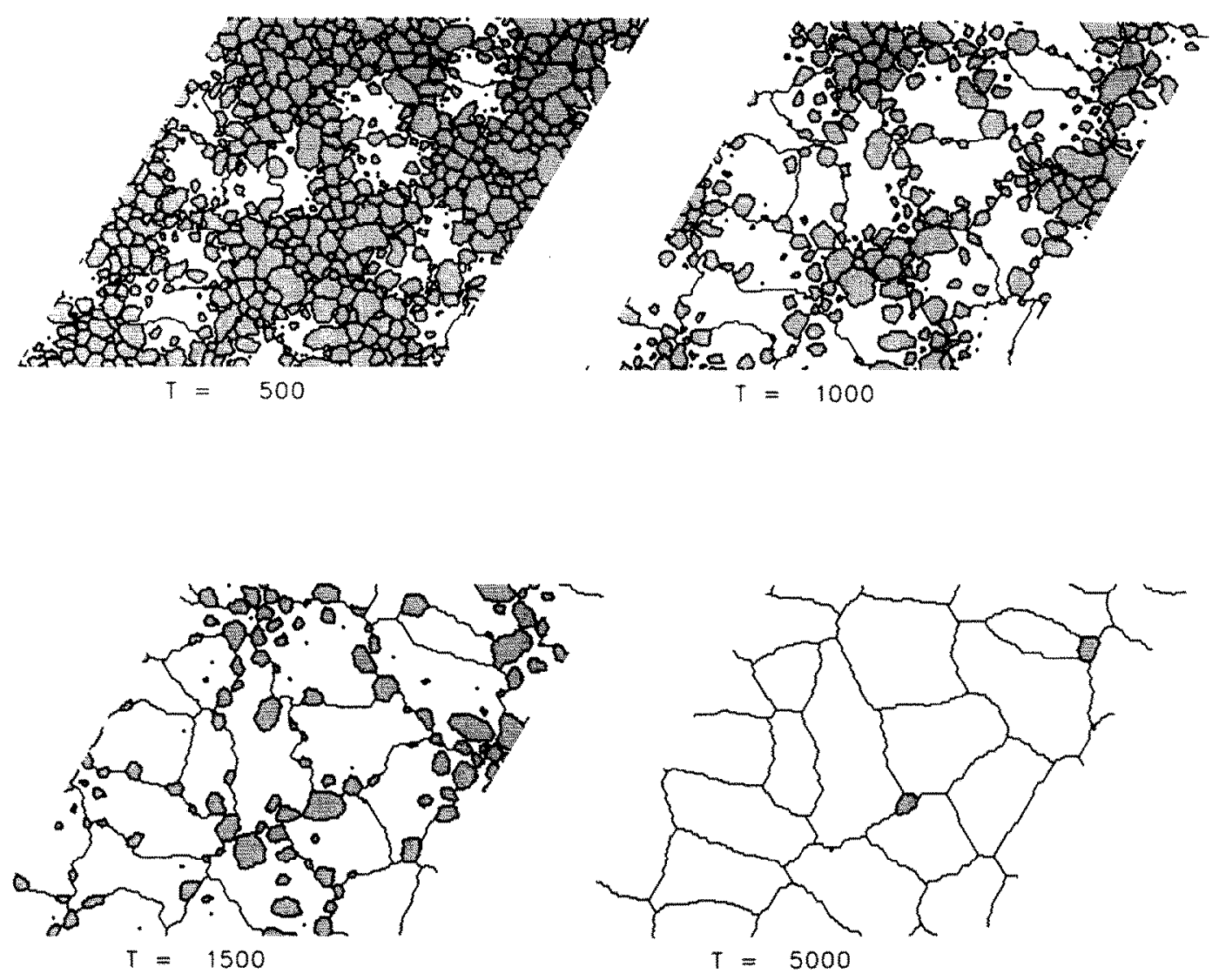

Fig. 6. Microstructural evolution under the conditions of anisotropic grain boundary energy shown in the previous figure with type I grains (unshaded) initially in the minority. At large times, the type I grains dominate and some grain coalescence occurs because of low effective $Q$.

$t^{2}$ and $\langle A\rangle$ increased as $t$ yielding an increase in the ratio $A_{1} /\langle A\rangle$ which is linear in time. For $\left.J_{1} / J_{2}\right\rangle 2$, the rate of increase of $A_{1} /\langle A\rangle$ increases with increasing anisotropy $\left(J_{1} / J_{2}\right)$.

To further illustrate the point that the kinetics are not those of normal grain growth, Fig. 11 plots the mean grain area vs time for the microstructures shown in Fig. 6. In this simulation, the ratio of grain boundary energies was 10 such that the Type I grains are initially in the minority but become the majority component. The slope of the plot of mean area vs time is slightly concave upwards instead of linear as is expected for normal grain growth [6].

A specific instance of the arrangement of boundary energies diagrammed in Fig. 2 is at the solid to liquid transition where the solid-liquid boundary has a lower energy than the solid-solid boundary. The behavior illustrated in Fig. 7 clearly models this situation. Even in the solid state it is possible for the arrangement of boundary energies diagrammed in Fig. 3 to arise if, for example, the misorientation between two different texture components is a special one such as the $38^{\circ}$ rotation about $\langle 111\rangle[21]$ as has been discussed for the growth of the cube component during recrystallization of rolled f.c.c. metals. Therefore the results obtained in this section for the simple scheme illustrated in Fig. 2 should be applicable to melting and a few special cases of textured materials. In general, however, a highly textured material might be expected to have low-angle-and therefore low energy - boundaries between grains of the same texture component and high-angle-therefore high energy - boundaries between grains of unlike component. The other special boundary character that must be considered, however, is that of mobility.

\section{ANISOTROPIC GRAIN BOUNDARY MOBILITIES}

All the Monte Carlo computer simulations of grain growth and abnormal grain growth that have been presented to date have assumed uniform mobility of the grain boundaries. In addition to the effects of anisotropic grain boundary energy considered in the previous section it is also possible to model the effects of variable mobility. Given a highly textured material consisting of two types of orientations (Type I and Type II), we define two classes of grain boundaries. The grain boundaries between grains of like type (Class 1) have low mobility, as low-angle boundaries are gencrally found to be experimentally, whereas the boundaries between grains of unlike type (Class 2) have high mobility, as found experimentally for 

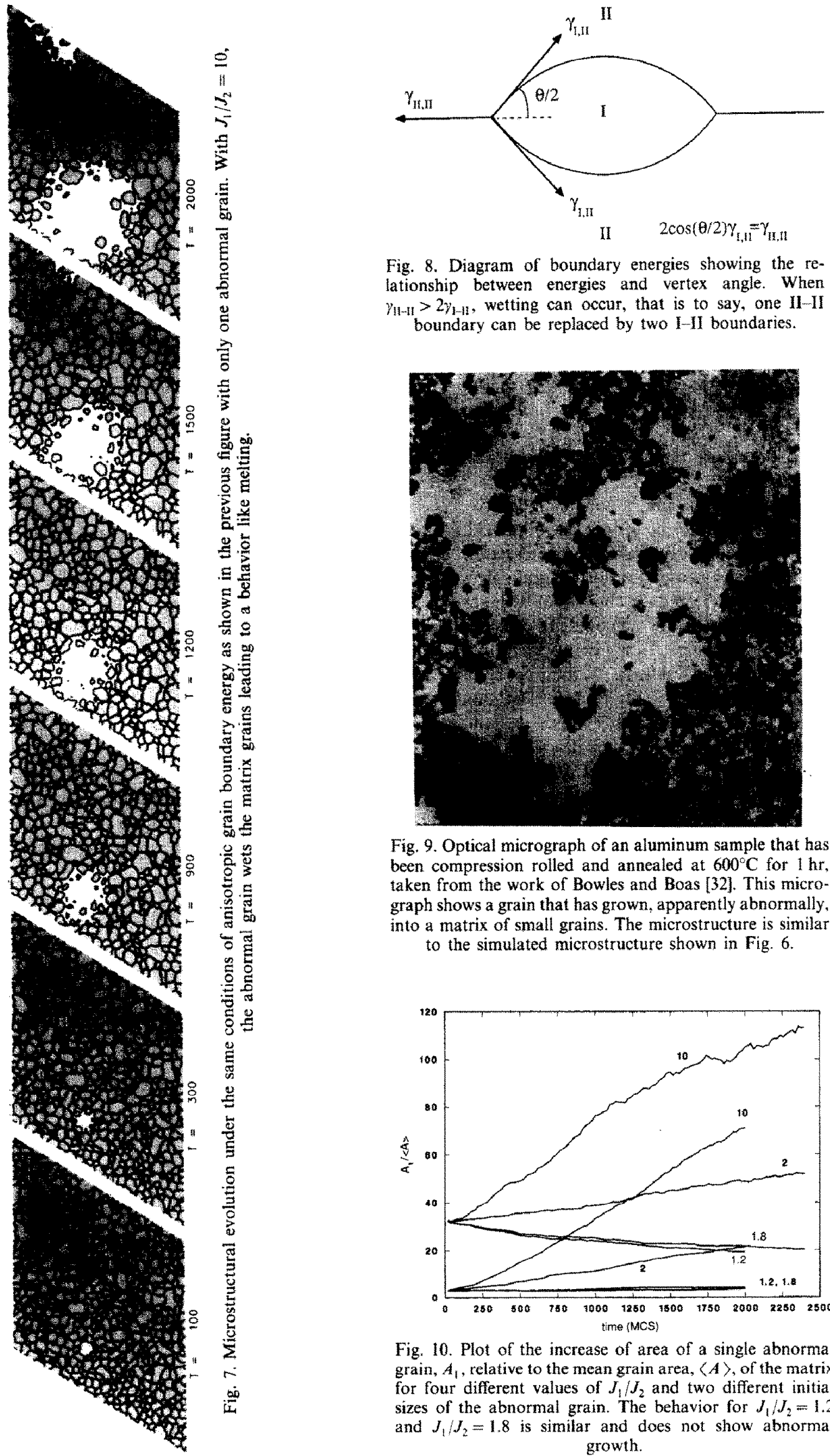

Fig. 8. Diagram of boundary energies showing the relationship between energies and vertex angle. When $\gamma_{1-11}>2 \gamma_{1-11}$, wetting can occur, that is to say, one II II boundary can be replaced by two $1-11$ boundaries.

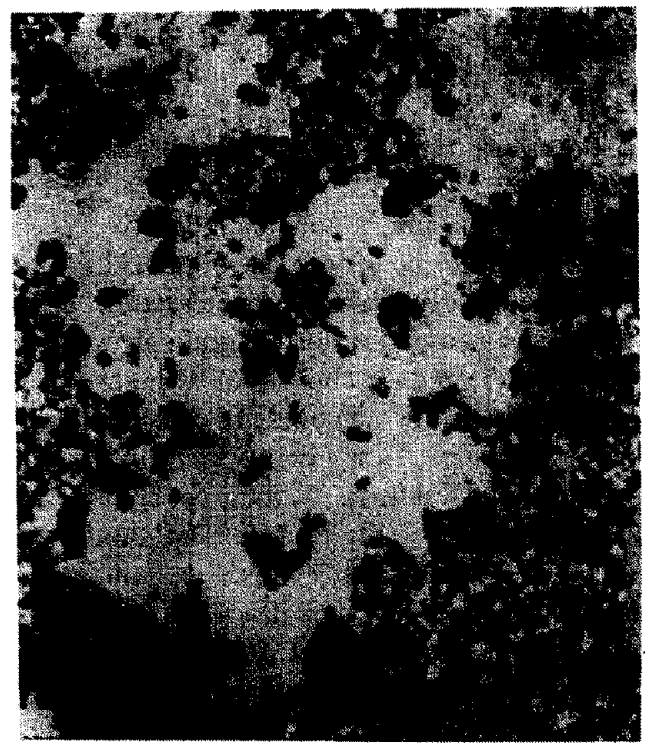

Fig. 9. Optical micrograph of an aluminum sample that has been compression rolled and annealed at $600^{\circ} \mathrm{C}$ for $1 \mathrm{hr}$, taken from the work of Bowles and Boas [32]. This micrograph shows a grain that has grown, apparently abnormally, into a matrix of small grains. The microstructure is similar to the simulated microstructure shown in Fig. 6.

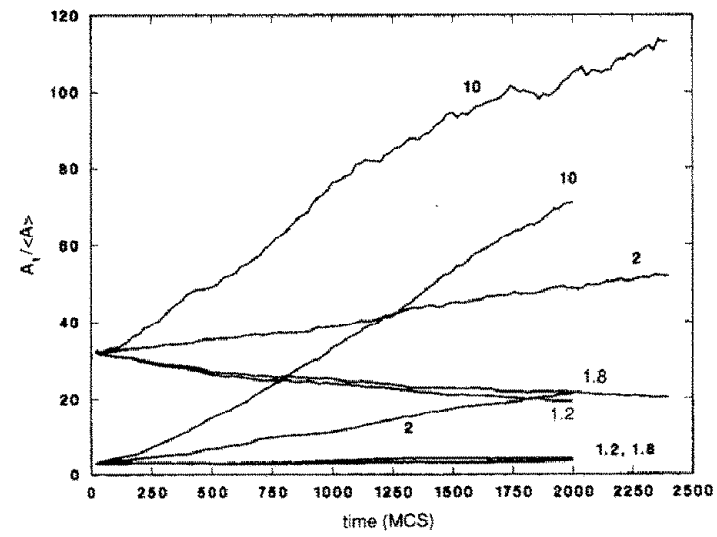

Fig. 10. Plot of the increase of area of a single abnormal grain, $A_{1}$, relative to the mean grain area, $\langle A\rangle$, of the matrix for four different values of $J_{1} / J_{2}$ and two different initial sizes of the abnormal grain. The behavior for $J_{1} / J_{2}=1.2$ and $J_{1} / J_{2}=1.8$ is similar and does not show abnormal growth. 


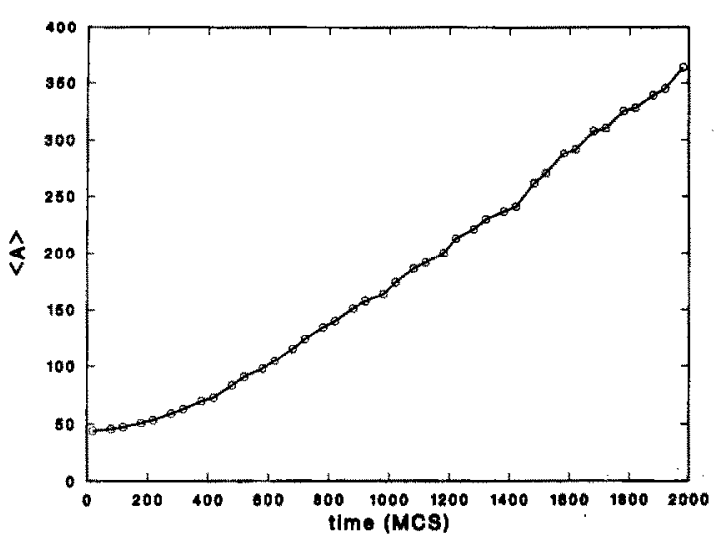

Fig. 11. Plot of $\langle A\rangle$ vs $t$ for the simulation illustrated in Fig. 6, showing faster than linear increase of mean area with time.

high-angle grain boundaries. This arrangement is illustrated by Fig. 12.

Anisotropic grain boundary mobility has been incorporated into the Monte Carlo model as follows. The energetics describing the interaction between lattice sites is as used for normal grain growth [i.e. as in equations (1) and (2) above, but with $J_{1}=J_{2}$ ]. The mobility difference is achieved by considering again two types of grains. Type I corresponds to $S_{i} \leq C$ and Type II corresponds to $S_{i}>C$, as before. When evaluating the possibility of a reorientation attempt, two classes of lattice site arise. A lattice site is considered to be of one class if it is adjacent to a I-II boundary which means that at least one of its six neighbors has an orientation that is of opposite type to that of the site itself. For this class of lattice site, a change of orientation is accepted with the same probability as for the normal model. That is, a reorientation attempt is accepted with probability, $p$, given by

$$
p=\left\{\begin{array}{cc}
e^{-\Delta E / k T} & \Delta E>0 \\
1 & \Delta E \leqslant 0
\end{array}\right.
$$

where $\Delta E$ is the encrgy change associated with the reorientation attempt.

The other class of lattice sites is that where all six neighbors of the site are of the same Type as the site itself (i.e. $S_{i}>C$ for the site and its six nearest neighbors, or $S_{i} \leqslant C$ for all seven sites). This class of site is either within a grain, adjacent to a I-I boundary or adjacent to a II-II boundary. For this class of site, a change in orientation is attempted with probability $1 / \mu^{*}$, where $\mu^{*}$ is the factor by which the mobility of the Type II-Type II or Type I-Type I grain boundaries is lower than that of Type I-Type II grain boundaries. Therefore for this class of site the probability that it reorients is

$$
p=\left\{\begin{array}{cc}
\frac{1}{\mu^{*}} e^{-\Delta E j k T} & \Delta E>0 \\
\frac{1}{\mu^{*}} & \Delta E \leqslant 0 .
\end{array}\right.
$$

This is considered equivalent to a reduction in the attempt frequency for reorientation. As before, the simulations were all performed at low temperatures such that orientation change attempts leading to an increase in system energy were not accepted.

For a sufficiently large difference in mobilities, a single large grain will grow faster than the mean matrix grain size. This statement is most easily understood by considering the limit of $\mu^{*}=\infty$ such that the matrix grain structure is pinned, i.e. the grain boundaries between matrix grains (Type II-Type II boundaries) have zero mobility. Then, provided the sign of the curvature on abnormal grain's boundaries are such as to make the abnormal grain grow, it can reach an arbitrarily large size relative to the matrix by simply allowing it enough time in which to grow. At the other extreme (i.e. $\mu^{*}=1$ ), on the other hand, it is known [10] that a large grain will grow less rapidly than the mean grain size.

Figure 13 shows the evolution of the microstructure for a simulation where the single (unshaded) Type I grain was placed in a matrix of Type II grains. The Class 1 boundaries, separating the Type I grain from the Type II matrix grains, had a mobility 7.5 times greater than that of the Class 2 boundaries between the matrix grains $\left(\mu^{*}=7.5\right)$. The abnormal grain clearly grows out into the matrix faster than the matrix coarsens. A notable difference between this microstructure and the simulations with anisotropic grain boundary energies (see Fig. 7) is that the boundary between the abnormal grain and the matrix is not rough, no occlusion of normal matrix grains occurs, and the abnormal/normal interface is well defined. This microstructure is closer to that commonly observed in experimental studies of abnormal grain growth.

In order to examine the kinetics of this type of abnormal grain growth, several simulations were performed with different mobility factors, ranging from 1.5 to 1000 . The results are plotted in Fig. 14 as relative radius, $\rho\left(=\left\{A_{1} /\langle A\rangle\right\}^{1 / 2}\right)$, vs time where $\langle A\rangle$ is calculated for the matrix grains only and does

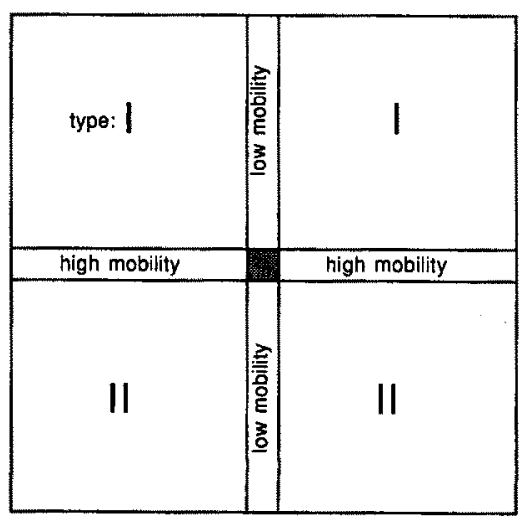

Fig. 12. Diagram of the arrangement of anisotropic grain boundary mobilities. 

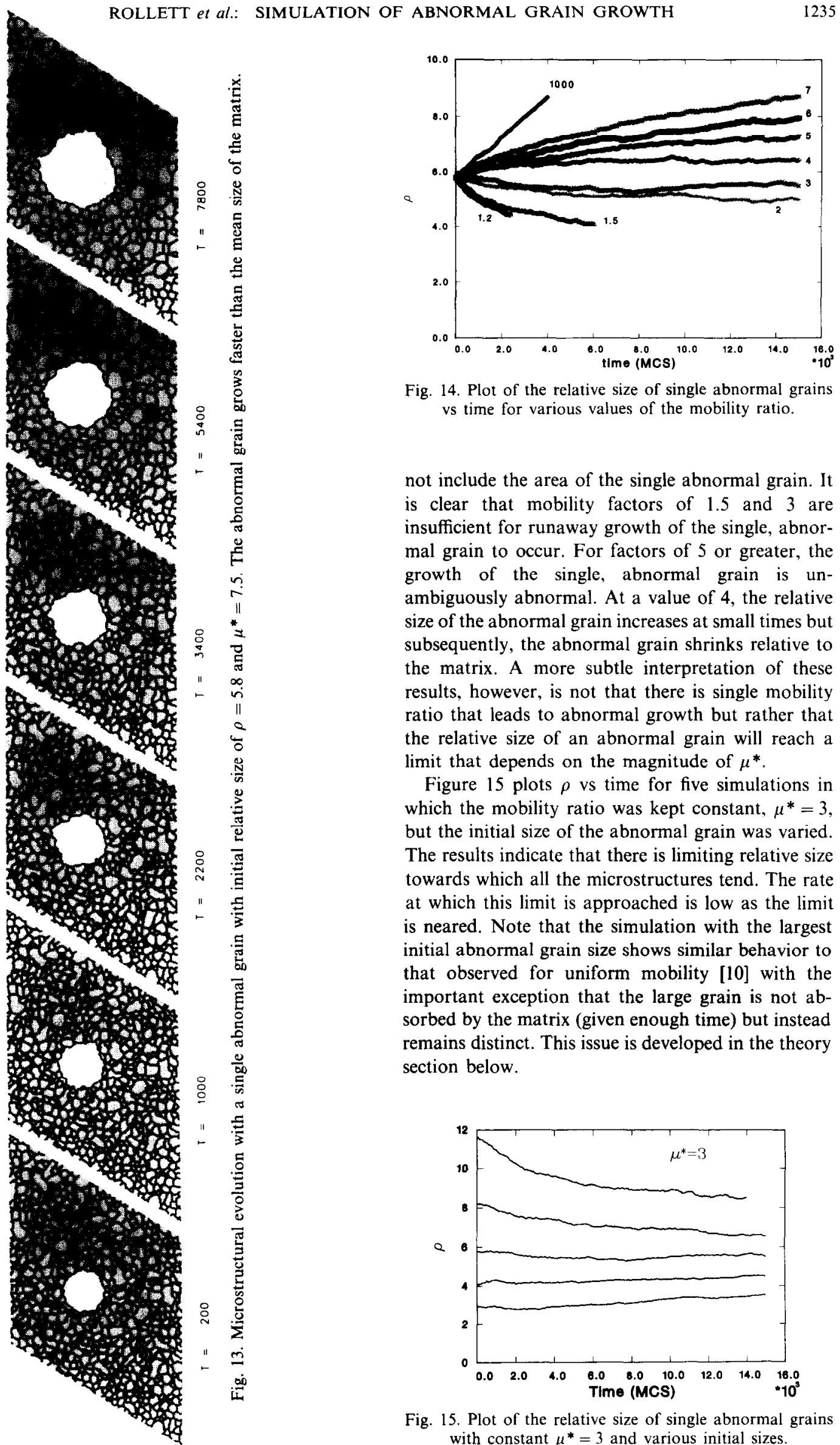

Fig. 14. Plot of the relative size of single abnormal grains vs time for various values of the mobility ratio.

not include the area of the single abnormal grain. It is clear that mobility factors of 1.5 and 3 are insufficient for runaway growth of the single, abnormal grain to occur. For factors of 5 or greater, the growth of the single, abnormal grain is unambiguously abnormal. At a value of 4 , the relative size of the abnormal grain increases at small times but subsequently, the abnormal grain shrinks relative to the matrix. A more subtle interpretation of these results, however, is not that there is single mobility ratio that leads to abnormal growth but rather that the relative size of an abnormal grain will reach a limit that depends on the magnitude of $\mu^{*}$

Figure 15 plots $\rho$ vs time for five simulations in which the mobility ratio was kept constant, $\mu^{*}=3$, but the initial size of the abnormal grain was varied. The results indicate that there is limiting relative size towards which all the microstructures tend. The rate at which this limit is approached is low as the limit is neared. Note that the simulation with the largest initial abnormal grain size shows similar behavior to that observed for uniform mobility [10] with the important exception that the large grain is not absorbed by the matrix (given enough time) but instead remains distinct. This issue is developed in the theory section below.

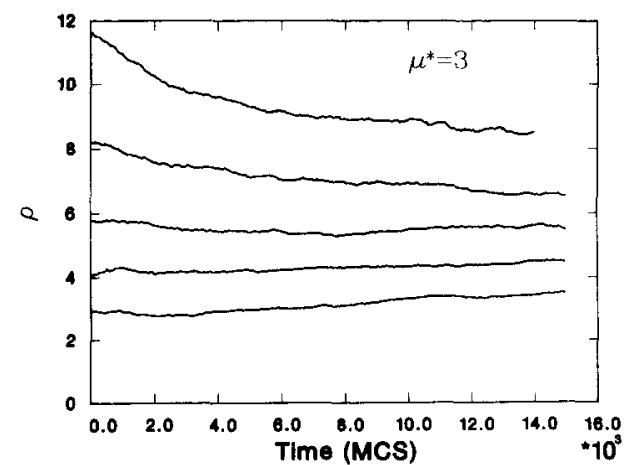

Fig. 15. Plot of the relative size of single abnormal grains with constant $\mu^{*}=3$ and various initial sizes. 


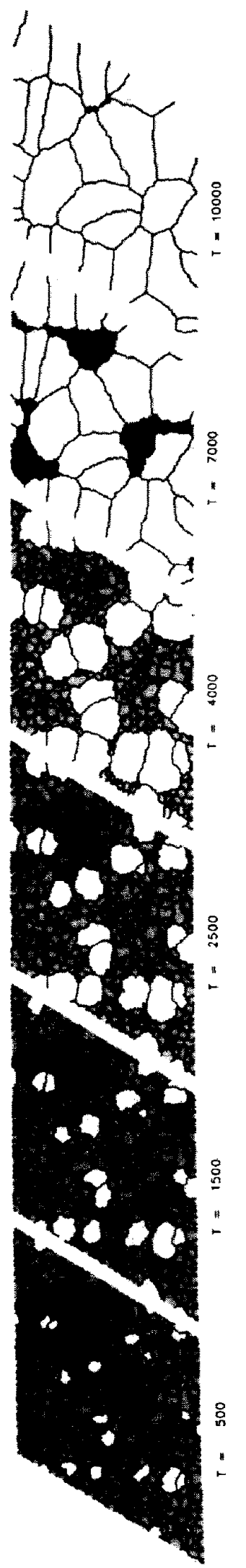

While the above, single abnormal grain simulations were useful for determining the degree of mobility anisotropy required for abnormal growth to occur, real microstructures have more than one abnormal grain. Simulations of abnormal grain growth in a more realistic situation with multiple abnormal grains have been performed. Figure 16 shows the microstructural evolution of a lattice where $C=20$ and $\mu^{*}=10$ was employed. The initial microstructure was the same as used above, i.e. the result of $1000 \mathrm{MCS}$ of normal grain growth except for the following modification. The orientation numbers of all grains were increased by 20 , giving $Q=68$ and then 20 grains, selected at random, were chosen to each have a unique orientation number from 1 to 20 . This modification of the initial microstructure guarantees that no coalescence of abnormal grains will occur despite the low effective $Q$ of the final microstructure once the normal grains have been eliminated.

Of the 20 abnormal grains that were initially present, only about seven were large enough and/or were in a favorable local environment that they grew. Those that grew, however, grew abnormally and rapidly dominated the microstructure. The temporal evolution of the mean grain size is shown in Fig. 17. Clearly the rate of growth of the mean area accelerates with time. The rate at which the microstructure transforms to the texture components of the abnormal grains can also be shown on a standard Johnson-Mehl-Avrami (JMA) plot as in Fig. 18 which shows the results of three simulations with the same initial microstructure described above and $\mu^{*}=10,100$ and 1000 . The data for $\mu^{*}=100$ and $\mu=1000$ are nearly coincident. The slope of the plots approaches 2 at long times which is the theoretically expected JMA exponent for site saturated nucleation conditions and two-dimensional growth.

Figure 19 shows the grain size distribution for the same simulation as a function of time; each distribution is normalized by the mean radius. The results show clearly how the distribution evolves from a near

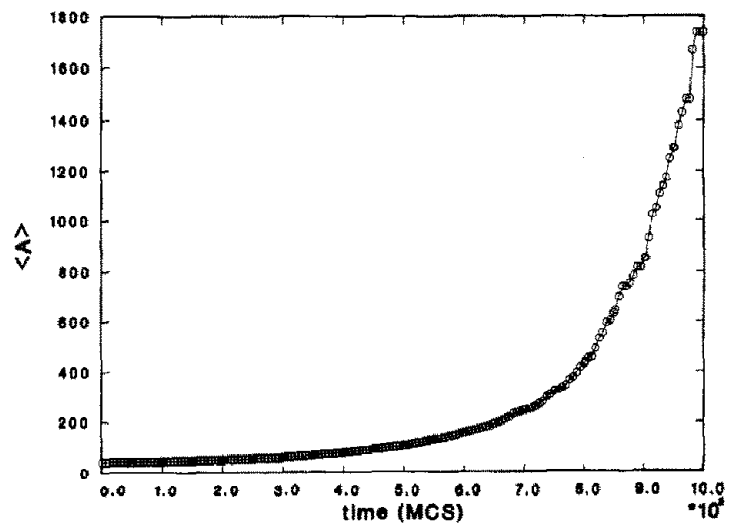

Fig. 17. Plot of the mean grain area (all grains) vs time for the simulation illustrated in the previous figure. 


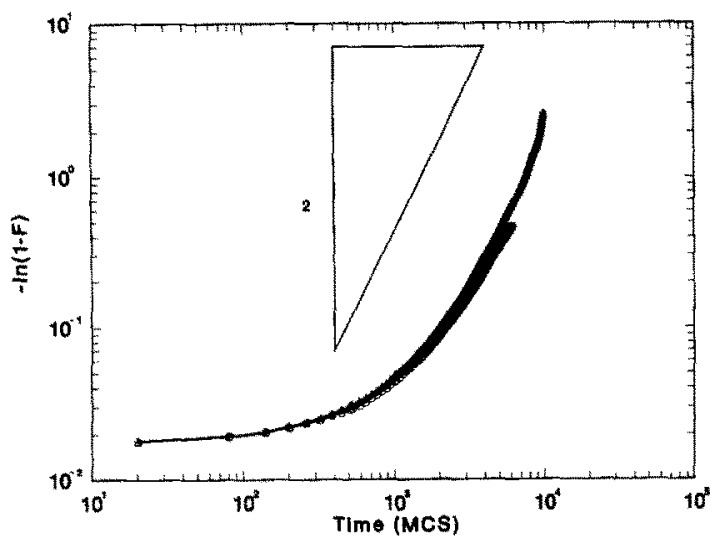

Fig. 18. JMA plot of the results of simulations of secondary recrystallization with 20 abnormal grains initially present and various values of the mobility ratio, $\mu^{*}=10,100$ and 1000. The results show that the slope of the plots approaches 2 at large times as expected theoretically for conditions of site saturated nucleation and two-dimensional growth.

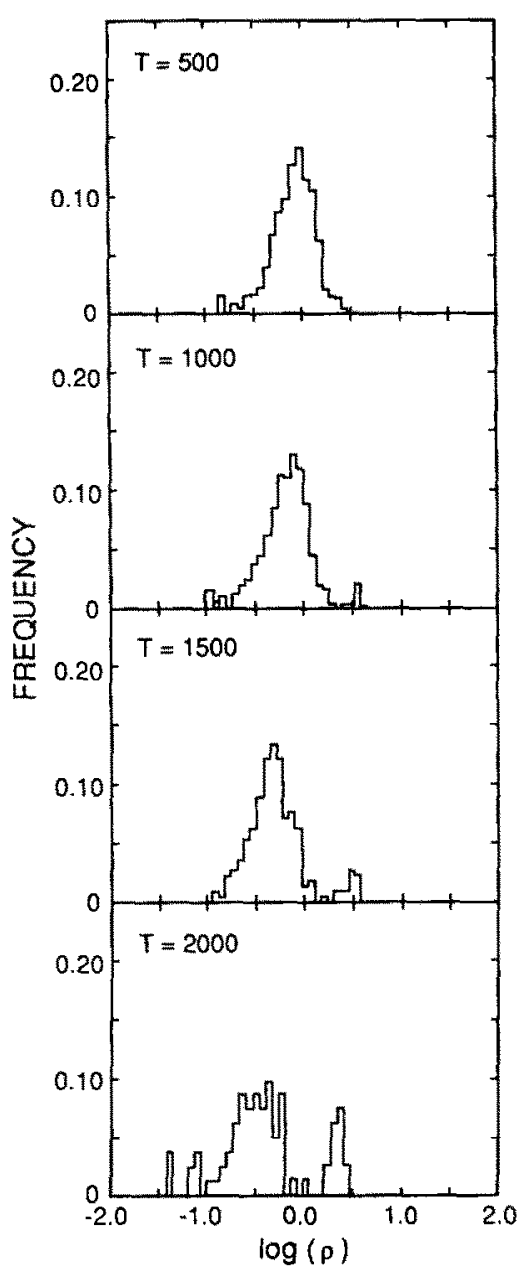

Fig. 19. Grain size distribution as a function of time $(T=500,1000,1500$ and $2000 \mathrm{MCS})$, plotted as volume fraction vs the logarithm of the relative radius. A normal grain size distribution at short times evolves into a bi-modal distribution at large times. Same simulation as for Fig. 16.
Rayleigh distribution $[5,6,31]$ at short times to a bi-modal distribution with peaks on either side of the mean at large times. The small lattice size ( 200 by 200 lattice points) and large mobility ratio $\left(\mu^{*}=10\right)$ chosen prevented the clear attainment of a bi-modal distribution.

\section{THEORY OF ABNORMAL GRAIN GROWTH}

In this section we develop a theory for the kinetics of abnormal grain growth as an extension of Hillert's [4] normal grain growth theory. We find that abnormal grain growth should be expected in this simple framework. The degree to which it occurs, however, is limited by the magnitude of the ratio, $\mu^{*}$, of the mobility of the boundaries separating the abnormal grains from the matrix grains to the mobility of the boundaries delimiting the matrix grains. This $\mu^{*}$ dependent limit on abnormal grain growth is then used to show that there is a minimum fraction of abnormal grains required for the complete secondary recrystallization of a material.

Hillert's theory of normal grain growth [4] employs the Lifshitz-Slyozov-Wagner (LSW) theory [33, 34] of interface controlled particle coarsening to obtain the grain size distribution as a function of time and grain size. The size distribution function is invariant with time and has a cut-off at large sizes. The mean grain radius, $\langle r\rangle$, obeys a power law relationship with time, $\langle r\rangle=c t^{1 / 2}$. Many experiments have demonstrated the validity of the power law relationship, albeit with exponents less than the 1/2 predicted by most theories. Recent simulation work has also shown that the exponent for grain growth in two and three dimensions $[6]$ is asymptotically $1 / 2$. The grain size distribution predicted by Hillert's theory [4] does not fit the available experimental and simulation data as well as the log-normal distribution [6]. Nevertheless we choose to work in the framework of Hillert's theory because it provides a simple, selfconsistent, and physically reasonable description of the process of normal grain growth. However, our main results are general in the sense that we believe they would not be significantly altered by the application of essentially any other self-consistent normal grain growth theory.

In what follows the relative growth rate of an abnormal grain, $\mathrm{d} \rho / \mathrm{d} t(\rho=r /\langle r\rangle$, where $r$ is the size of an individual abnormal grain), is derived as a function of the relative mobilities of the normal grains and the individual (abnormal) grain. The important result is that for mobility ratios greater than one, the abnormal grain can grow faster than the mean radius of the matrix of normal grains until a limiting size ratio is reached, $\rho^{\max }$. This upper limit is approximately proportional to the mobility ratio. This theorctical result is then compared to the results of the 2-D Monte Carlo simulations of abnormal grain growth presented in the previous section. 
Therefore the following discussion is limited to two dimensions though the results are generalizable to three dimensions.

Hillert [4] postulated the following equation for the mean growth rate of grains of size $r$

$$
\frac{\mathrm{d} r}{\mathrm{~d} t}=\alpha M \gamma\left(\frac{1}{r_{\mathrm{c}}}-\frac{1}{r}\right)
$$

where $r_{\mathrm{c}}$ is the critical radius at which grains neither shrink nor grow, $\alpha$ is a geometrical constant $(\alpha \approx 0.5)$, $M$ is the mobility of a grain boundary and $\gamma$ is the grain boundary free energy per unit area. Hillert [4] showed that $r_{\mathrm{c}}=\langle r\rangle$ for two dimensions by comparison with the equations of von Neumann [35] and Mullins [36] for the growth rate of a grain as a function of the number of its edges. Several justifications were provided [4] for the use of equation (5), including the observation that for grains very much larger than $r_{\mathrm{c}}$, the equation yields the expected limiting value for the growth rate. The growth rate of a grain as a function of its relative size $(\rho)$ may be derived from equation (5)

$$
\frac{\mathrm{d} \rho^{2}}{\mathrm{~d} t}=\frac{1}{\langle r\rangle^{2}}\left\{2 \alpha M \gamma(\rho-1)-\rho^{2} \frac{{ }^{2} \mathrm{~d}\langle r\rangle^{2}}{\mathrm{~d} t}\right\} .
$$

Hillert [4] points out that equation 6 yields a time invariant size distribution provided that

$$
\frac{\mathrm{d}\langle r\rangle^{2}}{\mathrm{~d} t}=0.5 \alpha M \gamma
$$

Choices other than that of equation (7) lead to situations in which either all grains are shrinking relative to the mean radius $(\mathrm{d} \rho / \mathrm{d} t<0$ for all $\rho$ ) or grains within a certain size range would be growing more rapidly than the mean. Both of these situation are clearly unacceptable for normal grain growth since they lead to time dependent grain size distributions. The latter case is, however, similar to the result derived below for abnormal grain growth where variable grain boundary mobilities are considered.

Hillert [4] showed from equations (6) and (7) that during normal grain growth the relative growth rate goes to zero not at $\rho=1(r=\langle r\rangle)$ but at $\rho=2$. This, then, is the upper grain size in the LSW size distribution. He stated erroneously that grains with $\rho>2$ would grow faster than the mean and would therefore be abnormal. Simulations by Srolovitz et al. [10] showed that abnormally large grains actually shrink relative to the mean radius. Thompson et al. [37] recently published an analysis demonstrating the absence of abnormal grain growth for $\rho>2$, using the LSW theory as applied to grain growth by Hillert.

Thompson et al. [37] derived the behavior of an abnormally large grain by introducing a second peak in the grain size distribution which was sharply peaked at the abnormal grain size. In the following discussion the same approach will be used since it closely approximates the anisotropic mobility simulations of the previous section. The crucial feature of our derivation is the introduction of a mobility ratio, $\mu^{*}$, between the abnormal grain and the matrix of normal grains. The radius of normal grains is denoted by a lower case $r$ whereas abnormal grains are indicated by an upper case $R$. The radius ratio $\rho$ is as previously defined.

For interface-controlled motion, the evolution of the grain size distribution, $f(r, t)$, is constrained [34] by the continuity requirement

$$
\frac{\partial f}{\partial t}=\frac{-2 \gamma M}{r_{\mathrm{c}}} \frac{\partial}{\partial r}\left\{\frac{\left(r-r_{\mathrm{c}}\right)}{r} f(r, t)\right\} .
$$

From this relation Wagner [34] derived a time invariant size distribution for which the temporal evolution of the critical radius is

$$
\langle r(t)\rangle^{2}-\langle r(0)\rangle^{2}=\gamma M t
$$

where $\langle r(0)\rangle$ is the mean radius at time zero and $\langle r\rangle=r_{\mathrm{c}}$ as before. Following Thompson et al. [37], consider the introduction of abnormal grains whose size is $R$ and whose mobility is larger than the normal grains by a factor $\mu^{*}$. The abnormal grains are represented by a narrowly peaked size distribution, $\Phi(R, t)$ which is added to the distribution of normal grains, $f(r, t)$. To find the temporal evolution of the abnormal grains with enhanced mobility boundaries, we substitute $R$ for $r$ in equation (8), noting that only this single radius value is of interest because of the assumed sharpness of $\Phi$ :

$$
\begin{aligned}
\frac{\partial \Phi(r-R(t))}{\partial t} & =\frac{-2 \gamma \mu^{*} M}{r_{\mathrm{c}}} \\
& \times \frac{\partial}{\partial r}\left\{\frac{\left(R(t)-r_{\mathrm{c}}\right)}{R(t)} \Phi[r-R(t)]\right\} .
\end{aligned}
$$

Given that $\partial[r-R(t)] / \partial t=-\mathrm{d} R / \mathrm{d} t$ and that $\partial[r-R(t)] / \partial r=1$, equation (10) can be simplified to

$$
\frac{\mathrm{d} R}{\mathrm{~d} t}=\frac{2 \gamma \mu * M}{r_{\mathrm{c}}} \frac{\left(R-r_{\mathrm{c}}\right)}{R}
$$

which reduces to Hillert's equation, equation (5), for uniform mobility (i.e. $\mu^{*}=1$ ). The variable of interest is the size of the abnormal grain relative to the mean grain size, $\rho=R /\langle r\rangle$, such that the relative growth rate, $\mathrm{d} \rho / \mathrm{d} t$, is given by

$$
\frac{\mathrm{d} \rho}{\mathrm{d} t}=\frac{\mathrm{d}}{\mathrm{d} t}\left(\frac{R}{\langle r\rangle}\right)=\frac{1}{\langle r\rangle^{2}}\left\{\langle r\rangle \frac{\mathrm{d} R}{\mathrm{~d} t}-R \frac{\mathrm{d}\langle r\rangle}{\mathrm{d} t}\right\}
$$

where $\langle r\rangle$ is assumed to be equal to $r_{c}$ as before. From equation (5) we find that $\mathrm{d}\langle r\rangle / \mathrm{d} t=\gamma M / 2\langle r\rangle$, such that

$$
\frac{\mathrm{d} \rho}{\mathrm{d} t}=\frac{1}{\langle r\rangle^{2}}\left\{2 \gamma \mu * M \frac{R-\langle r\rangle}{R}-R \frac{\gamma M}{2\langle r\rangle}\right\} .
$$

Rewriting equation (13) in terms of the relative grain size gives

$$
\frac{\mathrm{d} \rho}{\mathrm{d} t}=\frac{\gamma M}{2 \rho\langle r\rangle^{2}}\left\{4 \mu^{*}(\rho-1)-\rho^{2}\right\}
$$


This derivation assumes that the presence of the abnormal grains does not significantly perturb the evolution of normal grains far from the abnormal grains. As stated above, Thompson et al. [37] point out that the relative growth rate under uniform mobility conditions is negative for all values of $\rho$ except $\rho<2$.

Now consider the effect of an enhanced mobility of the abnormal grains, $\mu^{*}>1$. There is now a range of relative sizes over which the relative growth rate is positive and the abnormal grains can grow faster than the mean. The upper limit of relative size which should be reached is simply obtained by setting $\mathrm{d} \rho / \mathrm{d} t=0$ in equation (14)

$$
\rho^{\max }=2\left\{\mu^{*}+\sqrt{\mu^{*}\left(\mu^{*}-1\right)}\right\}
$$

where we have taken the larger of the two solutions of the quadratic equation. For a mobility ratio of 4 , equation (15) predicts that abnormal grains will grow until they are approximately 14.9 times larger than the mean radius of the normal matrix grains. Conversely, if a grain for which $\rho>14.9$ were present in the system, it would grow less rapidly than the mean radius of the matrix until it too was 14.9 times larger than the mean. Once the abnormal grains all reach $\rho^{\text {max }}$ it would appear that the size distribution would remain time invariant even though it would be far from the normal one. This leads to the theoretical possibility of having a time invariant size distribution whose shape is markedly different from the normal as a result of the presence of a range of grain boundary mobilities in the material. The bimodal distribution illustrated in Fig. 19 is an example of the type of grain size distribution that could be observed.

Turning to a comparison with the simulation results, there is qualitative agreement in that both theory and simulation indicate that grains with a mobility advantage can grow abnormally and that there is an upper limit to the size ratio, $\rho^{\max }$, that is dependent on the mobility ratio. Quantitatively, however, it appears that the simple theory based on Hillert's theory [4] predicts a limit that increases more rapidly with $\mu^{*}$ than found in simulation. Referring to the simulations of Fig. 15 where $\mu^{*}=3$, a reasonable value for $\rho^{\max }$ would appear to be approximately 6 whereas equation (15) suggests a value of 10.9 . This suggests that more sophisticated theories of grain growth should be examined for possible adaptation to mobility variations.

The concept of an upper limit to the relative size of abnormal grains for a given mobility ratio is of consequence in secondary recrystallization. It is possible to make a very simple estimate of the mobility ratio required for complete secondary recrystallization by assuming that $C \rho^{\max }$, where $C=O(1)$, would need to be approximately one half of the mean spacing of abnormal grains. Defining $I$ as the number fraction of abnormal grains (equal to the volume fraction of abnormal grains if the abnormal grains have the same initial mean size as the matrix),

$$
I \approx\left(2 \rho^{\max }\right)^{-d} \text { for } d \text { dimensions. }
$$

As a numerical example, if only 1 in $10^{6}$ grains were abnormal, a mobility ratio of 13 would suffice to produce complete secondary recrystallization in three dimensions. Such a ratio is not unreasonable in light of the mobility ratios measured by Aust and Rutter [22]. A more accurate calculation of these parameters would of course need to take into account the evolution of the normal matrix during secondary recrystallization and the effects of impingement of the abnormal grains.

\section{SUMMARY}

i. Monte Carlo computer simulations have shown that abnormal grain growth can occur by two distinct mechanisms. Both of these mechanisms are related to anistropy in the properties of grain boundaries, namely anisotropic grain boundary energy and anisotropic grain boundary mobility.

ii. Microstructures obtained from the model of anisotropic grain boundary energies show that the abnormal grains wet and dissolve the matrix grain boundaries. This leads to a characteristic occlusion of matrix grains behind the advancing abnormal grain. This unusual microstructure has been observed experimentally.

iii. Microstructures obtained following abnormal grain growth in the model of anisotropic grain boundary mobility show normal microstructures with the exception of the grain size distribution. These microstructures correspond very well to those commonly observed for abnormal grain growth or "secondary recrystallization".

iv. Theoretical arguments show that when a distribution of grain boundary mobilities is present in the material, abnormal grain growth can occur. The abnormal grain cannot, however grow to arbitrary size but is limited to a maximum size (relative to the mean grain size) which scales with the magnitude of the mobility anisotropy.

Acknowledgements - The authors are grateful to Professor to R. D. Doherty for criticism of the manuseript and to Professor F. Spaepen for fruitful discussions. The work was supported in part by the U.S. Department of Energy under contract number W-7405-ENG-36.

\section{REFERENCES}

1. F. R. Rhines and K. R. Craig, Metall. Trans, 5, 413 (1974).

2. G. F. Bolling and W. C. Winegard, Acta metall. 6, 283 (1958).

3. J. P. Drolet and A. Galibois, Acta metall. 16, 1387 (1968).

4. M. Hillert, Acta metall. 13, 227 (1965).

5. N. P, Louat, Acta metall, 22, 721 (1974).

6. M. P. Anderson, G. S. Grest and D. J. Srolovitz, Phil. $M$ ag. $B$ In press. 
7. D. J. Srolovitz, R. Eykholt, D. Barnett and J. P. Hirth, Phys. Rev. B 35, 6107 (1987).

8. D. J. Srolovitz and G. N. Hassold, Phys, Rev. B 35, 6902 (1987).

9. W. W. Mullins, J. appl. Phys. 59, 1341 (1986).

10. D. J. Srolovitz, G. S. Grest and M. P. Anderson, Acta metall. 33, 2233 (1986)

11. D. J. Srolovitz, G. S. Grest and M. P. Anderson, Acta metall. 34, 1833 (1986); D. J. Srolovitz, G. S. Grest, M. P. Anderson and A. D. Rollett, Acta metall. 36, 2115 (1988).

12. C. J. Simpson, K. T. Aust and W. C. Winegard, Metall. Trans. 2, 987 (1971).

13. G. Riontino, C. Antonione, L. Battezzati, F. Marino and M. C. Tabasso, J. Mater. Sci. 14, 86 (1979).

14. C. Antonione, L. Battezzati, A. Lucci, G. Riontino and M. C. Tabasso, J. Mater. Sci. 15, 1730 (1980).

15. C. Antonione, F. Marino, G. Riontino and M. C. Tabasso, J. Mater. Sci. 12, 747 (1977).

16. C. Antonione, G. dellaGatta, G. Riontino and G. Venturello, J. Mater. Sci. 8, 1 (1973).

17. J. J. Petrovic and L. J. Ebert, Metall. Trans. 3, 1131 (1972).

18. J. J. Petrovic and L. J. Ebert, Metall. Trans. 3, 1123 (1972).

19. J. Harase, R. Shimizu and T. Watanabe, Eighth Int. Conf. on Textures of Materials (ICOTOM-8), (edited by J. S. Kallend and G. Gottstein), p. 723. Metall. Soc. (1988).

20. J. E. May and D. Turnbull, Trans. metall. Soc. A.I.M.E. 212, 769 (1958)

21. K. Lucke, Proc. ICOTOM-7, Holland, p. 195 (1984).
22. K. T. Aust and J. W. Rutter, in Recovery and Recrystallization of Metals, p. 13I. T.M.S.-A.I.M.E., New York (1962)

23. G. Abbruzzese and K. Lucke, Proc. 7th Int. Riso Conf. un Annealing Processes - Recovery, Recrystallization and Grain Growth, Riso, p. 1 (1986).

24. G. Abbruzzese and K. Lucke, Acta. metall. 34, 905 (1986).

25. W. T. Read and W. Shockley, Phys. Rev. 78, 275 (1950).

26. G. S. Grest, D. J. Srolovitz and M. P. Anderson, Acta metall. 33, 509 (1985).

27. J. E. Burke, Trans. Am. Inst. Min. Metall. Petr. Engrs 180, 73 (1945).

28. H. Makita, S. Hanada and O. Izumi, Acta metall. 36, 403 (1988).

29. W. Brickenkamp and K. Lücke, Proc. ICOTOM-6, Tokyo, p. 570 (1981); W. Brickenkamp, thesis, RWTH, Aachen (1983).

30. M. P. Anderson, D. J. Srolovitz, G. S. Grest and P. S. Sahni, Acta metall. 32, 783 (1984).

31. D. I. Srolovitz, M. P. Anderson, P. S. Sahni and G. S. Grest, Acta metall. 32, 793 (1984).

32. J. S. Bowles and W. Boas, J. Inst. Metals 74, 501 (1948).

33. I M. Lifshitz and V. V. Slyozov, J. exp. theoret. Phys. 21, 479 (1958), in Russian.

34. C. Wagner, Z. Elektrochem. 65, 581 (1961).

35. J. von Neumann. Metal Interfaces, p. 108. Am. Soc. Metals, Cleveland, Ohio (1952).

36. W. W. Mullins, J. appl. Phys. 27, 900 (1956).

37. C. V. Thompson, H. J. Frost and F. Spaepen, Acta metall. 35, 887 (1987). 